\title{
Влияние внутренних оптических потерь на генерацию стимулированного излучения в среднем ИК диапазоне в волноводных гетероструктурах с квантовыми ямами $\mathrm{HgCdTe} / \mathrm{CdHgTe}$
}

\author{
(C) В.В. Уточкин ${ }^{1}$, А.А. Дубинов ${ }^{1}$, М.А. Фадеев ${ }^{1}$, В.В. Румянцев ${ }^{1}$, Н.Н. Михайлов ${ }^{2}$, \\ C.A. Дворецкий ${ }^{2}$, В.И. Гавриленко ${ }^{1}$, С.В. Морозов ${ }^{1,3, \text { व }}$ \\ ${ }^{1}$ Институт фризики микроструктур Российской академии наук, \\ 603950 Нижний Новгород, Россия \\ ${ }^{2}$ Институт физики полупроводников Сибирского отделения Российской академии наук, \\ 630090 Новосибирск, Россия \\ ${ }^{3}$ Нижегородский государственный университет им. Н.И. Лобачевского, \\ 603950 Нижний Новгород, Россия \\ ฯ E-mail: more@ipmras.ru
}

Поступила в Редакцию 12 апреля 2021 г.

В окончательной редакции 19 апреля 2021 г.

Принята к публикации 19 апреля 2021 г.

\begin{abstract}
Исследованы волноводные гетероструктуры с массивом из 10 квантовых ям $\mathrm{HgCdTe} / \mathrm{CdHgTe,} \mathrm{выращенные}$ в рамках одной технологической серии и рассчитанные на генерацию стимулированного излучения в диапазоне длин волн 20-30 мкм. В „коротковолновой“ структуре получено стимулированное излучение на длине волны $\sim 23.9$ мкм при температуре $10 \mathrm{~K}$, в то время как в „длинноволновой“ стимулированное излучение не наблюдалось. Проведены расчеты оптического поглощения в пассивных слоях для обеих структур, продемонстрировано, что в ,длинноволновой“ структуре его уровень выше, и предлагаются подходы по минимизации его влияния на генерацию стимулированного излучения.
\end{abstract}

Ключевые слова: средний ИК диапазон, $\mathrm{HgCdTe,} \mathrm{квантовые} \mathrm{ямы,} \mathrm{стимулированное} \mathrm{излучение.}$

DOI: $10.21883 /$ FTP.2021.10.51445.49

\section{1. Введение}

Компактные источники когерентного излучения дальнего инфракрасного (ИК) и терагерцового (ТГц) диапазонов востребованы для многочисленных задач спектроскопии газов и твердых тел, медицины и экологии. Лучшие характеристики в данных диапазонах имеют квантово-каскадные лазеры (ККЛ) на основе $\mathrm{A}^{\mathrm{III}} \mathrm{B}^{\mathrm{V}}$ полупроводников. В ККЛ уже получена генерация в диапазонах 3-25 мкм [1] и 60-300 мкм [2]. Однако ККЛ не покрывают спектральный диапазон 25-60 мкм, в котором наблюдается сильное фононное поглощение в $\mathrm{A}^{\mathrm{III}} \mathrm{B}^{\mathrm{V}}$ материалах.

Альтернативой ККЛ в данном диапазоне могут служить межзонные полупроводниковые лазеры с квантовыми ямами (КЯ) $\mathrm{HgCdTe} / \mathrm{CdHgTe}$ В подобных длинноволновых лазерах безызлучательная оже-рекомбинация играет важную роль, так как при продвижении в дальний ИК диапазон скорость оже-рекомбинации в них многократно возрастает за счет уменьшения ее пороговой энергии [3], и оже-рекомбинация может являться основным механизмом межзонной рекомбинации $[4,5]$. Фактором, эффективно повышающим пороговую энергию оже-рекомбинации, является [квази]симметричность законов дисперсии носителей. Так, в случае симметричных гиперболических законов дисперсии дираковских фермионов законы сохранения не могут быть выпол- нены при любой начальной энергии частиц, участвующих в оже-процессе [6]. Гетероструктуры с узкими КЯ $\mathrm{HgCdTe} / \mathrm{CdHgTe} \mathrm{являются} \mathrm{одной} \mathrm{из} \mathrm{полупроводниковых}$ систем, в которой можно достичь квазигиперболического закона дисперсии в окрестности Г-точки при ненулевой ширине запрещенной зоны и тем самым подавить оже-рекомбинацию $[7,8]$.

Значительное развитие процедуры роста $\mathrm{HgCdTe}$ структур методом молекулярно-пучковой эпитаксии (МПЭ), произошедшее за последние два десятилетия, сделало возможным получение гетероструктур с массивами КЯ высокого оптического качества с малой остаточной концентрацией примесей и дефектов [9]. Усовершенствование технологии роста обусловило прогресс в разработке межзонных $\mathrm{HgCdTe}$ лазеров дальнего ИК диапазона, что подтверждается экспериментальными результатами. Так, в недавней работе в таких структурах было получено стимулированное излучение (СИ) с длиной волны 19.5 мкм при температуре $18 \mathrm{~K}$ [10].

Цель настоящей работы - исследование факторов, влияющих на возможность получения СИ в волноводных структурах с квантовыми ямами $\mathrm{HgCdTe} / \mathrm{CdHgTe}$, pacсчитанных на генерацию в длинноволновом диапазоне от 20 мкм и более. В частности, исследуется влияние оптических потерь в пассивных слоях на примере двух структур, рассчитанных на длину волны генерации 24 и 27 мкм. В работе исследуются спектры фотолюминес- 
ценции (ФЛ) структур при комнатной температуре и низкотемпературного СИ при длинноволновой оптической накачке, а также приводятся результаты расчета величины оптических потерь для дизайна диэлектрического волновода, реализованного в исследуемых структурах.

\section{2. Методика эксперимента}

Обе исследованные структуры были выращены в рамках одной технологической серии методом молекулярнопучковой эпитаксии на полуизолирующей $\operatorname{GaAs}(013)$ подложке с буферами из ZnTe (50 нм) и CdTe (10 мкм) с in situ эллипсометрическим контролем состава и толщины слоев. Ex situ характеризация структур проводилась при помощи экспресс-методики, описанной в работе [11]. Структура 1 содержала в активной области массив из 10 КЯ $\mathrm{Hg}_{0.935} \mathrm{Cd}_{0.065} \mathrm{Te} / \mathrm{Cd}_{0.61} \mathrm{Hg}_{0.39} \mathrm{Te}$, структура 2 массив из $10 \mathrm{KЯ} \mathrm{Hg}_{0.95} \mathrm{Cd}_{0.05} \mathrm{Te} / \mathrm{Cd}_{0.7} \mathrm{Hg}_{0.3}$ Те. Для эффективной локализации основной ТЕ моды в обеих структурах массив КЯ был выращен в волноводном слое $\mathrm{Hg}_{0.25} \mathrm{Cd}_{0.75}$ Те толщиной $\sim 9$ мкм.

Естественно, выколотые грани образцов, выращенных на подложках $\operatorname{GaAs}(013)$, не образуют резонатор Фабри-Перо из-за специфики кристаллографической ориентации, поэтому в работе исследовалось СИ при однопроходовом усилении. При исследовании спектров ФЛ и СИ образцы монтировались на холодный палец криостата замкнутого цикла Advanced Research Systems DE-202 с рабочим диапазоном температур 8-300 K. Излучение от образцов заводилось в фурье-спектрометр Bruker Vertex 80v, работавший в режиме пошагового сканирования. Оптическое возбуждение при исследовании спектров ФЛ осуществлялось непрерывным диодным лазером $\left(\lambda_{\mathrm{ex}}=808 \mathrm{нм}\right)$ со средней мощностью $360 \mathrm{MBT,} \mathrm{а}$ при исследовании СИ - импульсным $\mathrm{CO}_{2}$-лазером (длина волны 10.6 мкм, длительность импульса 100 нс, частота повторения 5-20 Гц, энергия в импульсе до 40 мДж). Пятно $\mathrm{CO}_{2}$-лазера полностью покрывало поверхность исследованных образцов. В качестве приемника в низкотемпературных экспериментах использовался кремниевый болометр, охлаждаемый жидким гелием (спектральный диапазон чувствительности $20-650 \mathrm{~cm}^{-1}$ ), а в экспериментах при комнатной температуре использовался HgCdTe приемник Kolmar Technologies D317.

\section{3. Результаты и обсуждение}

Перед исследованием излучения при низких температурах для обоих образцов проводилась характеризация методом спектроскопии ФЛ при комнатной температуре и возбуждении 808 нм диодным лазером. На рис. 1 приведены полученные спектры ФЛ обеих структур.

Видно, что полная ширина на уровне половинной амплитуды (FWHM) спектров при комнатной температуре практически одинакова и составляет порядка $400 \mathrm{~cm}^{-1}$. Согласно данным работы [11], в структурах с

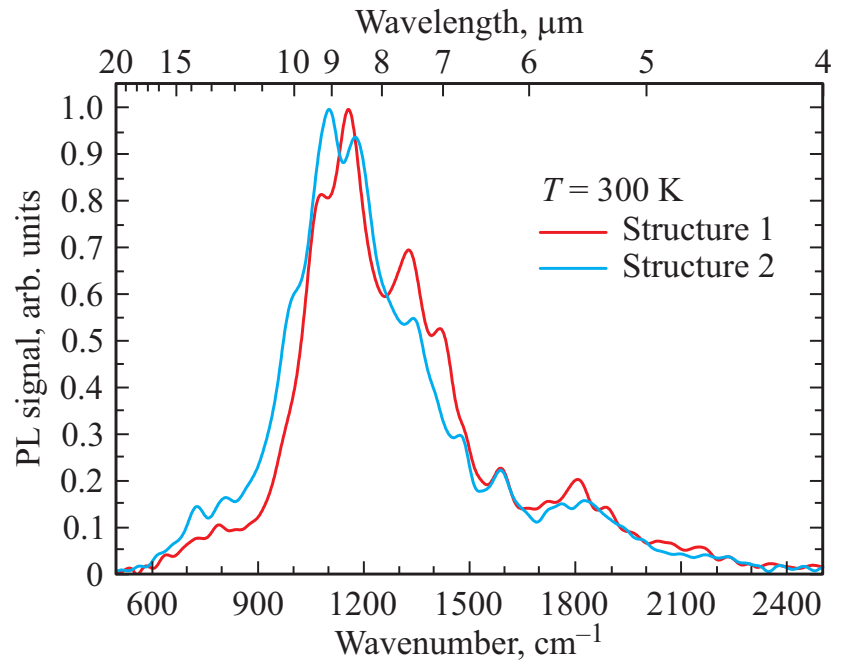

Рис. 1. Спектры спонтанной фотолюминесценции обоих образцов при комнатной температуре и оптическом возбуждении на длине волны 808 нм.

КЯ $\mathrm{HgCdTe/CdHgTе} \mathrm{высокого} \mathrm{оптического} \mathrm{качества,} \mathrm{рас-}$ считанных на диапазон 10-20 мкм, FWHM спектров ФЛ при комнатной температуре составляет 400-500 $\mathrm{cm}^{-1}$. В таких структурах удается получить СИ при относительно низких пороговых интенсивностях возбуждения. В той же работе [11] приводится эмпирическая закономерность, позволяющая оценить спектральное положение линии СИ при криогенных температурах по спектру ФЛ при $300 \mathrm{~K}$. При условии возникновения в структуре СИ при температурах, близких к температуре жидкого гелия, его длина волны отстоит от положения длинноволнового края спектра комнатной ФЛ на полувысоте на $\sim 600 \mathrm{~cm}^{-1}$ в длинноволновую сторону. Положение точки, расположенной на полувысоте длинноволнового края спектра ФЛ, для „коротковолновой“ структуры 1 составляет $\sim 1020 \mathrm{~cm}^{-1}$, а для ,длинноволновой“ структуры $2-\sim 970 \mathrm{~cm}^{-1}$. Таким образом, ожидалось, что положение линии СИ при $10 \mathrm{~K}$ для первой структуры будет находиться вблизи $420 \mathrm{~cm}^{-1}(\lambda \sim 23.6$ мкм $)$, а для второй $-370 \mathrm{~cm}^{-1}(\lambda \sim 26.9$ мкм $)$.

Далее при $T=10 \mathrm{~K}$ было проведено исследование спектров излучения в обоих образцах при оптическом возбуждении импульсным $\mathrm{CO}_{2}$-лазером. Эксперимент проводился в оптимальной для наблюдения СИ геометрии, мощность лазера менялась вплоть до максимальной мощности установки. В то время как в „коротковолновой“ структуре 1 удалось получить СИ на длине волны 23.9 мкм с относительно низкой пороговой мощностью накачки (см. рис. 2), в „длинноволновой“ структуре 2 наблюдался лишь широкий спектр спонтанного излучения, по интенсивности близкий к фоновому излучению даже при максимальной мощности лазерного возбуждения.

Известно, что базовым условием развития лазерной генерации является превышение величины усиления над общими потерями в структуре, что выражается 


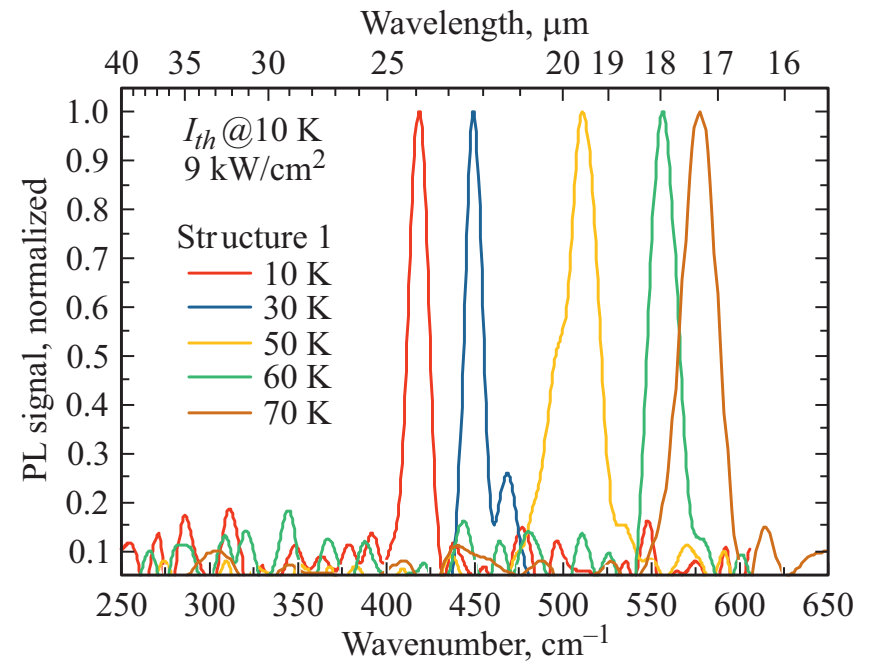

Pис. 2. Спектры СИ структуры 1 при различных температуpax при оптическом возбуждении импульсным $\mathrm{CO}_{2}$-лазером. (Цветной вариант рисунка представлен в электронной версии статьи).

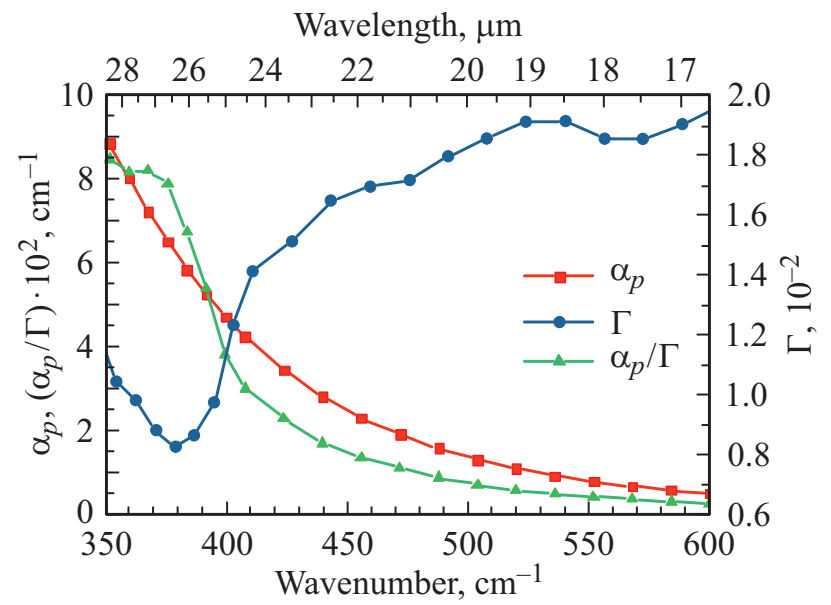

Рис. 3. Рассчитанные частотные зависимости параметров $\alpha_{p}, \Gamma$ и $\alpha_{p} / \Gamma$ для исследованных структур. (Цветной вариант рисунка представлен в электронной версии статьи).

соотношением

$$
\left(G-\alpha_{a}\right) \Gamma=\alpha_{p}+\alpha_{m}
$$

где $G$ - коэффициент усиления активной среды, $\alpha_{a}-$ коэффициент потерь в активной среде, $\Gamma-$ фактор оптического ограничения, $\alpha_{p}$ и $\alpha_{m}-$ коэффициенты поглощения в пассивных слоях и потерь на зеркалах соответственно. При рассмотрении генерации СИ важен учет как внутренних потерь в волноводе $\alpha_{p}$ (при этом мы ограничивались рассмотрением режима однопроходного усиления излучения, пренебрегая потерями на зеркалах $\alpha_{m}$ ), так и фактора оптического ограничения. Для нахождения распределения электрического поля ТЕ-моды, определения фактора оптического ограничения, эффек- тивного показателя преломления и коэффициента поглощения $\alpha_{p}$ для волноводных мод проводилось численное решение уравнений Максвелла [12,13]. Диэлектрические проницаемости нелегированных GaAs и CdTe аппроксимировались на основании экспериментальных данных, представленных в работе [14]. В отсутствие экспериментальных данных по частотной зависимости диэлектрической проницаемости слоев $\mathrm{Hg}_{0.25} \mathrm{Cd}_{0.75}$ Те в диапазоне длин волн 20-30 мкм было использовано приближение, в котором действительная часть диэлектрической проницаемости принималась равной 8.1 [15], а мнимая часть считалась такой же, как в $\mathrm{CdTe}$. Рассчитанные частотные зависимости $\alpha_{p}, \Gamma$ и $\alpha_{p} / \Gamma$ для обеих структур (так как волноводы у них практически одинаковые) представлены на рис. 3.

Как видно из рис. 3, в рассматриваемом волноводе потери, связанные с поглощением в пассивных слоях, быстро растут, а фактор оптического ограничения уменьшается при продвижении в длинноволновую область. Так, параметр $\alpha_{p} / \Gamma$ в диапазоне длин волн 26-28 мкм приблизительно на порядок больше, чем на $\lambda \sim 18$ мкм. Наблюдаемый рост параметра $\alpha_{p} / \Gamma$ связан с тем, что в рассматриваемом диапазоне длин волн находится область остаточных лучей в GaAs, играющего роль подложки для структур. С ростом длины волны происходит одновременное увеличение показателя преломления и коэффициента поглощения в $\mathrm{GaAs}$. Когда показатель преломления GaAs в длинноволновой области начинает превышать показатель преломления буферного (и ограничительного для моды) слоя CdTe, происходит „вытекание моды“ в подложку, приводящее к увеличению коэффициента потерь $\alpha_{p}$ и уменьшению фактора оптического ограничения Г. Из рис. 3 видно, что параметр $\alpha_{p} / \Gamma$ для структуры 2 на предполагаемой длине волны генерации СИ при $10 \mathrm{~K} \lambda_{\mathrm{SE}}=26.9$ мкм в 4 раза больше, чем для структуры 1 на ее длине волны генерации СИ при $10 \mathrm{~K} \lambda_{\mathrm{SE}}=23.9$ мкм. Следовательно, для возникновения СИ в структуре 2 необходим, как минимум, в 4 раза больший коэффициент усиления в КЯ, что требует кратного увеличения концентрации фотовозбужденных носителей в ней. Отсутствие наблюдения СИ в структуре 2 говорит о том, что такое увеличение концентрации фотовозбужденных носителей недостижимо из-за оже-рекомбинации [5].

Для подавления влияния растущего параметра $\alpha_{p} / \Gamma$ можно применить три подхода. Первый из них состоит в росте более толстого ограничивающего слоя $\mathrm{CdTe}$, либо волноводного слоя $\mathrm{HgCdTe}$, что позволит уменышить проникновение моды в GaAs-подложку и связанное с этим поглощение, а также увеличить фактор оптического ограничения Г [16]. Однако этот подход требует соответствующего увеличения времени роста структуры, что представляется трудновыполнимым для роста структур на диапазон 25-30 мкм.

Второй подход - уменьшение за счет сильного легирования показателя преломления GaAs подложки до значений, меньших величины показателя преломления 
CdTe-буферного слоя [16]. При этом нет необходимости в росте толстых ограничивающего слоя СdTe и волноводного слоя $\mathrm{HgCdTe}$, но подход требует разработки технологии роста $\mathrm{HgCdTe-гетероструктур} \mathrm{на} \mathrm{легированных}$ подложках GaAs.

Третий подход состоит в увеличении числа КЯ в структуре. Коэффициент поглощения излучения $\mathrm{CO}_{2}$-лазера накачки исследованными структурами составляет единицы процентов, так как излучение поглощается только в массиве КЯ, а коэффициент поглощения одиночной КЯ $<1 \%$ [17]. Увеличение количества КЯ в структуре увеличит как фактор оптического ограничения, так и эффективность длинноволновой накачки при той же плотности мощности оптического возбуждения, что позволит скомпенсировать рост потерь в пассивных слоях без увеличения времени роста структуры или перехода на новый тип подложки.

\section{4. Заключение}

В данной работе проведено исследование ФЛ и СИ при оптической накачке двух волноводных структур с массивом из 10 КЯ $\mathrm{HgCdTe} / \mathrm{CdHgTe}$. В более „коротковолновой“ структуре с КЯ $\mathrm{Hg}_{0.935} \mathrm{Cd}_{0.065} \mathrm{Te} / \mathrm{Cd}_{0.61} \mathrm{Hg}_{0.39} \mathrm{Te}$ было получено СИ на 23.9 мкм при $T=10 \mathrm{~K}$ при пороговой интенсивности накачки 9 КВт/см ${ }^{2}$. В более „длинноволновой“ структуре 2 с КЯ $\mathrm{Hg}_{0.95} \mathrm{Cd}_{0.05} \mathrm{Te}_{2} / \mathrm{Cd}_{0.7} \mathrm{Hg}_{0.3} \mathrm{Te}$ СИ не наблюдалось при любой интенсивности оптического возбуждения. Обнаружено, что параметр $\alpha_{p} / \Gamma$, где $\alpha_{p}-$ коэффициент поглощения в пассивных слоях, а $Г-$ фактор оптического ограничения, резко возрастает при продвижении в диапазон остаточных лучей в GaAs и является основным фактором, препятствующим генерации СИ в структуре 2. Предложены три подхода для минимизации влияния данного фактора - переход к дизайнам лазерных структур с большей толщиной волноведущего либо ограничивающего слоя, рост на легированной подложке, а также увеличение числа КЯ в активной области структуры, что является наиболее удобным решением с технологической точки зрения.

\section{Финансирование работы}

Расчет внутренних потерь в пассивных слоях исследованных структур проведен при поддержке Российского фонда фундаментальных исследований (грант № 20-52-50004), а характеризация образцов и исследование СИ проведено при поддержке Российского научного фонда (грант № 17-12-01360).

\section{Благодарности}

В работе использовано оборудование УСУ „Фемтоспектр“ ЦКП ИФМ РАН.

\section{Конфликт интересов}

Авторы заявляют, что у них нет конфликта интересов.

\section{Список литературы}

[1] M.S. Vitiello, G. Scalari, B. Williams, P. De Natale. Opt. Express, 23 (4), 5167 (2015).

[2] B.S. Williams. Nature Photonics, 1 (9), 517 (2007).

[3] V.V. Utochkin, K.E. Kudryavtsev, M.A. Fadeev, A.A. Razova, D.S. Bykov, V.Ya. Aleshkin, A.A. Dubinov, N.N. Mikhailov, S.A. Dvoretsky, V.V. Rumyantsev, V.I. Gavrilenko, S.V. Morozov. Laser Phys., 31, 015801 (2021).

[4] V. Rumyantsev, M. Fadeev, V. Aleshkin, N. Kulikov, V. Utochkin, N. Mikhailov, S. Dvoretskii, S. Pavlov, H.-M. Hübers, V. Gavrilenko, C. Sirtori, Z.F. Krasilnik, S.V. Morozov. Phys. Status Solidi B, 256, 1800546 (2019).

[5] V. Ya. Aleshkin, V.V. Rumyantsev, K.E. Kudryavtsev, A.A. Dubinov, V.V. Utochkin, M.A. Fadeev, G. Alymov, N.N. Mikhailov, S.A. Dvoretsky, F. Teppe, V.I. Gavrilenko, S.V. Morozov. J. Appl. Phys., 129, 133106 (2021).

[6] G. Alymov, V. Vyurkov, V. Ryzhii, A. Satou, D. Svintsov. Phys. Rev. B, 97, 205411 (2018).

[7] M. Orlita, D.M. Basko, M.S. Zholudev, F. Teppe, W. Knap, V.I. Gavrilenko, N.N. Mikhailov, S.A. Dvoretskii, P. Neugebauer, C. Faugeras, A-L. Barra, G. Martinez, M. Potemski. Nature Physics, 10, 233 (2014).

[8] G. Alymov, V. Rumyantsev, S. Morozov, V. Gavrilenko, V. Aleshkin, D. Svintsov. ACS Photonics 7, 98 (2019).

[9] N.N. Mikhailov, R.N. Smirnov, S.A. Dvoretsky, Yu.G. Sidorov, V.A. Shvets, E.V. Spesivtsev, S.V. Rykhlitski. Int. J. Nanotechnology, 3 (1), 120 (2006).

[10] S.V. Morozov, V.V. Rumyantsev, M.A. Fadeev, M.S. Zholudev, K.E. Kudryavtsev, A.V. Antonov, A.M. Kadykov, A.A. Dubinov, N.N. Mikhailov, S.A. Dvoretsky, V.I. Gavrilenko. Appl. Phys. Lett., 111, 192101 (2017).

[11] С.В. Морозов, В.В. Уточкин, В.В. Румянцев, М.А. Фадеев, А.А. Разова, В.Я. Алешкин, В.И. Гавриленко, Н.Н. Михайлов, С.А. Дворецкий. Письма ЖТФ, 47 (3), 51 (2021).

[12] Л.Д. Ландау, Е.М. Лифшиц. Электродинамика сплошных сред (М., Наука, 1989).

[13] H.C. Casey, M.B. Panich. Heterostructure lasers (N.Y., Academic Press, 1978).

[14] E.D. Palik. Handbook of optical constants of solids (Orlando, Academic Press, 1985).

[15] A. Rogalski. Rep. Progr. Phys., 68, 2267 (2005).

[16] А.А. Дубинов, В.В. Румянцев, М.А. Фадеев, В.В. Уточкин, С.В. Морозов. ФТП, 55, 455 (2021).

[17] V.Ya. Aleshkin, A.A. Dubinov, V.V. Rumyantsev, M.A. Fadeev, O.L. Domnina, N.N. Mikhailov, S.A. Dvoretsky, F. Teppe, V.I. Gavrilenko, S.V. Morozov. J. Phys.: Condens. Matter, 30, 495301 (2018).

Редактор А.Н. Смирнов 


\section{Effect of internal optical losses \\ on stimulated emission generation \\ in the mid-IR range in waveguide \\ heterostructures with $\mathrm{HgCdTe} / \mathrm{CdHgTe}$ quantum wells}

V.V. Utochkin ${ }^{1}$, A.A. Dubinov ${ }^{1}$, M.A. Fadeev ${ }^{1}$,

V.V. Rumyantsev' ${ }^{1}$, N.N. Mikhailov' ${ }^{2}$, S.A. Dvoretsky ${ }^{2}$,

V.I. Gavrilenko ${ }^{1}$, S.V. Morozov ${ }^{1,3}$

${ }^{1}$ Institute for Physics of Microstructures,

Russian Academy of Sciences,

603950 Nizhny Novgorod, Russia

${ }^{2}$ Institute of Semiconductor Physics,

Siberian Branch Russian Academy of Sciences,

630090 Novosibirsk, Russia

${ }^{3}$ Lobachevsky State University of Nizhny Novgorod,

603950 Nizhny Novgorod, Russia

Abstract We study two heterostructures with an array of 10 $\mathrm{HgCdTe} / \mathrm{CdHgTe}$ quantum wells, grown within a single technological series and designed to generate stimulated emission (SE) in the $20-30 \mu \mathrm{m}$ wavelength range. In the more short-wavelength structure we obtain SE at the wavelength $\lambda \sim 23.9 \mu \mathrm{m}$ at $10 \mathrm{~K}$, while no SE was obtained in the more long- wavelength structure. We calculate optical absorption in passive layers for both structures and show it to be higher in the more long-wavelength structure. Finally, we consider several ways to minimize absorption influence on SE generation.

Продолжение публикации материалов Симпозиума см. в № 11. 